[0212-7199 (2005) 22: 3; pp 118-123] ALES DE MEDICINA INTERN Copyright (C) 2005 ARAN EDICIONES, S.L

AN. MED. INTERNA (Madrid) Vol. 22, N. ${ }^{\circ}$ 3, pp. 118-123, 2005

\title{
Evolución de la incidencia de neumonías en la Comunidad Valenciana desde 1995 a 2001. Estudio retrospectivo
}

\author{
A. M. COMES CASTELlANO, J. A. LLUCH RODRIGO, A. PORTERO ALONSO, \\ E. PASTOR VILLALBA, M. SANZ VALERO
}

Centro Consultor en Vacunología. Centro Superior de Investigaciones en Salud Pública. Dirección General de Salud Pública. Consellería de Sanidad de Valencia

DEVELOPMENT OF THE INCIDENCE OF PNEUMONIAS IN THE AUTONOMOUS COMMUNITY OF VALENCIA THROUGHOUT THE 1995-2001 PERIOD. A RETROSPECTIVE STUDY

\section{RESUMEN} 2001

Fundamento: Conocer la evolución de las neumonías desde 1995 a

Método: Los datos se obtuvieron del Conjunto Mínimo Básico de Datos, seleccionando tres causas de hospitalización por neumonías en la Comunidad Valenciana, bronconeumonía, neumonía por organismo no especificado y neumonía neumocócica. El análisis estadístico se realizó mediante el programa SPSS, hallando las incidencias de las neumonías y su evolución en el tiempo y observando interacciones entre las variables género, edad, estancia y procedencia.

Resultados: La incidencia fue de 209 por 100.000 habitantes, aumentando significativamente a lo largo del periodo hasta estabilizarse a partir de 1999. La neumonía afecta más a hombres que a mujeres, sobre todo en mayores de 65 años. Por edades, son más frecuentes en menores de 5 años y en mayores de 65, concentrándose el mayor número de casos en 76-77 años. Se han observado diferencias estadísticamente significativas en las incidencias por provincias. El número de estancias es mayor en hombres que en mujeres y aumentan por grupos de edad, siendo más elevadas en los mayores de 65 años. La estancia media, varía según la edad, sin existir diferencias significativas entre género o grupo de edad.

Conclusiones: La neumonía, patología originada por diferentes causas, afecta más a hombres que a mujeres, a menores de 5 años y a mayores de 65. Las incidencias se estabilizan en los últimos años de estudio. Se deberían seguir estas investigaciones para dilucidar si las diferentes neumonías en este periodo, evolucionan igualmente y si se dan interacciones entre las variables estudiadas

PALABRAS CLAVE: Neumonía. Streptococcus pneumoniae. Incidencia. Estancia media. Hospitalización por neumonía.

\section{ABSTRACT}

Aim: To know development pneumonias throughout 1995 to 2001.

Methods: The data source was obtained from the database of the Minumum Basic Data Set. We selected three pneumonia hospitalized causes in the Community of Valencia, Broncopneumonia, and Pneumonia for not specified organism and invasive pneumococcal disease. It was realized the statistical analysis by SPSS programme. Calculating the pneumonia incidence rate and its time development and observing the interactions between age group, genre, mean of age, stay in the hospital and origin.

Results: The incidence for the period was 209 per 100.000 people, that increased significantly until it stabilized in 1999. The pneumonia affected more frequently men than women and specially aged 65 years or more. For age the pneumonias are more frequent in subjects younger than 5 and older than 65, (maximum 76-77 years old). It was observed statistically significant differences between provinces. The stays on hospital was greater for men and this ground with age groups, this is longer in people 65 years old or older. The average stay depending on the aged groups but do not exist statistically differences between genre and age group.

Conclusions: Pneumonia is a pathology originated from different causes, it affect principally men and more especially at younger than 5 years and older than 65 years. The incidences stabilizing on the studied period last years. This works must be continued for clarify if it is the same way for the development by the different pneumonias types on this time period and if exists the interactions between the different variables.

KEY WORDS: Pneumonia. Streptococcus pneumoniae. Incidence. Average stay. Hospitalization by pneumonia.

Comes Castellano AM, Lluch Rodrigo JA, Portero Alonso A, Pastor Villalba E, Sanz Valero M. Evolución de la incidencia de neumonías en la Comunidad Valenciana desde 1995 a 2001. Estudio retrospectivo. An Med Interna (Madrid) 2005; 22: 118-123.

\section{INTRODUCCIÓN}

La Organización Mundial de la Salud (OMS) considera la infección neumocócica como una de las enfermedades de origen bacteriano más importantes de las sociedades occidentales (1).

La mayor parte de las infecciones neumónicas son causadas por Streptococcus pneumoniae del que solo conocemos parte de su estructura. Si que se sabe que solo los neumococos capsulados son capaces de ocasionar enfermedades, debido a la actividad antifagocitaria de la cápsula. En la pared celular se encuentran los determinantes antigénicos que definen al grupo, el polisacárido $\mathrm{C}$ y las proteínas $\mathrm{M}$ y $\mathrm{A}$. Se conocen alrededor de 90 serogrupos capsulados con distinta virulencia, en función de su capacidad para producir toxinas y enzimas. Por otro lado, la frecuencia relativa de los diferentes serogru- 
pos varia en función del tiempo y del área geográfica. En nuestro país, sólo 16 de los 90 serotipos, causan el 85\% de las enfermedades neumocócicas y los serogrupos más frecuentes son los siguientes: 19, 6, 23, 3, 14 y 9, que representan el $60 \%$ del total de serogrupos estudiados (2).

Las infecciones neumocócicas son más frecuentes en la población infantil (menor de 5 años) y en la población mayor de 65 años así como en aquellas personas que poseen factores de riesgo tales como, afecciones bronco pulmonares, enfermedades coronarias crónicas e infecciones de las vías respiratorias.

Según datos del CDC (Center for Disease Control and Prevention) en EE.UU., se producen de 2.600 a 6.200 casos de meningitis causadas por S. pneumoniae, de 150.000 a 570.000 casos de neumonía y 7 millones de casos de otitis media $(3,4)$. La mortalidad estimada es de 40.000 muertes anuales siendo el $90 \%$ en personas de avanzada edad. La incidencia varia según el país y el grupo de población, siendo de 280 casos por 100.000 personas mayores de 75 años (5).

La incidencia de neumonías en España $(2,6)$ se encuentra en torno a los 420 casos por 100.000 habitantes y la tasa de mortalidad por neumonías por cualquier germen, es de 16/100.000 habitantes/año. La máxima incidencia de las infecciones neumocócicas tiene lugar en los niños menores de 2 años por la incapacidad de responder a los antígenos polisacáridos debido a la inmadurez de su sistema inmune, la incidencia de la enfermedad invasiva por $S$. pneumoniae en esta población, menores de 2 años en Cataluña, es de 61 casos/100.000 (7), siendo la incidencia de meningitis neumocócica de 7-9 casos/100.000 habitantes. Según el EPINE (Estudio de Prevalencia de Infecciones Nosocomiales) en España, durante el año 1999, las infecciones respiratorias comunitarias por el neumococo fueron el 9,9 y las nosocomiales el 3,3\% (8). En el año 2000 se produjeron un total de 7.590 muertes por neumonía, el 19,9 fueron hombres y el 18,1 mujeres por cada 100.000 habitantes, lo cual representa una tasa bruta de 21,6 de cada 10.000 fallecidos (9).

En la Comunidad Valenciana (datos del Registro de Mortalidad), la mortalidad por neumonías, independientemente del germen causante, supone una tasa bruta de entre un $16 \mathrm{y}$ un 19 por 100.000 , lo que equivale aproximadamente a un $2 \%$ de mortalidad proporcional. Se observa que en el año 1995 fallecieron 664 personas por neumonía, un 17,2/100.000 (y una mortalidad proporcional de 1,95) y tanto el número de fallecimientos como la tasa bruta o la mortalidad proporcional, van aumentando a lo largo del periodo de estudio hasta alcanzar valores para la mortalidad proporcional de 2,1 en el año 1996 o casi un millar de muertes en 1999 con una tasa bruta de 23,8/100.000.

El objeto de este trabajo, es estimar la evolución de las neumonías atendidas en los hospitales de la Comunidad Valenciana desde 1995 hasta el año 2001, a partir de la información recogida en el CMBD (Conjunto Mínimo Básico de
Datos) de los hospitales públicos de la Comunidad Valenciana, considerando que la patología atendida, por esta causa, en el medio hospitalario refleja de manera suficiente lo que sucede en la población general.

\section{MATERIAL Y MÉTODO}

A partir de la información recogida en la base de datos del CMBD correspondientes a los hospitales públicos de la Comunidad Valenciana de los años comprendidos entre 1995 y 2001 se han seleccionado aquellos registros en los que aparecía como diagnóstico al alta, los códigos de la CIE-9 486 (neumonía, organismo no especificado), 481 (neumonía neumocócica) y 485 (bronconeumonía, organismo no especificado) en cualquiera de los campos diagnósticos.

Cada registro se ha clasificado como neumonía neumocócica cuando aparecía este diagnóstico en alguno de los campos, como neumonía por organismo no especificado si no aparecía el código 481 y sí el 486, en cualquiera de los campos diagnósticos y como bronconeumonía en el resto de los casos.

Se ha analizado la incidencia en función de las variables edad, género, residencia (provincia), y días de estancia para todo el periodo y para cada uno de los años de los que se disponía información. Sólo se han considerado los registros válidos, en cada caso.

Para el análisis se han definido los grupos siguientes: G1: población de 0-5 años, G2: población de 6-14 años, G3: población de 15-45 años, G4: población de 46-64 años y G5: población mayor o igual a 65 años. Calculamos la media de edad para cada uno de los grupos definidos.

Los denominadores utilizados para el cálculo de las tasas se han obtenido del Instituto Nacional de Estadística (INE) (9), estimándose los datos para los años 1.995 y 1.997 ya que no hay datos oficiales para estos años.

El análisis de los resultados se ha realizado mediante el programa SPSS, versión 11.0 para Windows y Excel.

\section{RESULTADOS}

Se obtuvieron un total de 59.718 casos válidos para todo el periodo, $37.517(62,8 \%)$ fueron hombres y $22.201(37,2 \%)$ mujeres, con edades comprendidas entre 0-100 años y con una media de edad de 56,68 años, y una desviación estándar (D.s.) de 28,97 .

La incidencia de todos los diagnósticos de neumonía en la Comunidad Valenciana, desde el año 1995 hasta el 2001, es de $209 / 100.000$ siendo su intervalo de confianza al $95 \%$ (IC) de $\pm 1,68$, oscilando entre $151 \pm 3,82$ correspondiente al año 1995 y $241 \pm 4,78$ correspondiente al año 1999 (Tabla I, Fig. 1).

TABLA I

INCIDENCIAS GLOBALES DURANTE CADA AÑO DEL PERIODO DE ESTUDIO

\begin{tabular}{lcccccccc}
\hline & 1995 & 1996 & 1997 & 1998 & 1999 & 2000 & 2001 & Total \\
\hline Hombres & 192 & 239 & 254 & 271 & 306 & 303 & 306 & 268 \\
Mujeres & 110 & 135 & 146 & 151 & 179 & 169 & 173 & 152 \\
Total & 151 & $186^{*}$ & $199^{*}$ & $210^{*}$ & $241^{*}$ & 235 & 238 & 209 \\
\hline
\end{tabular}

$\left({ }^{*}\right)$ años en que el incremento de las incidencias, con respecto al año precedente es estadísticamente significativo. 


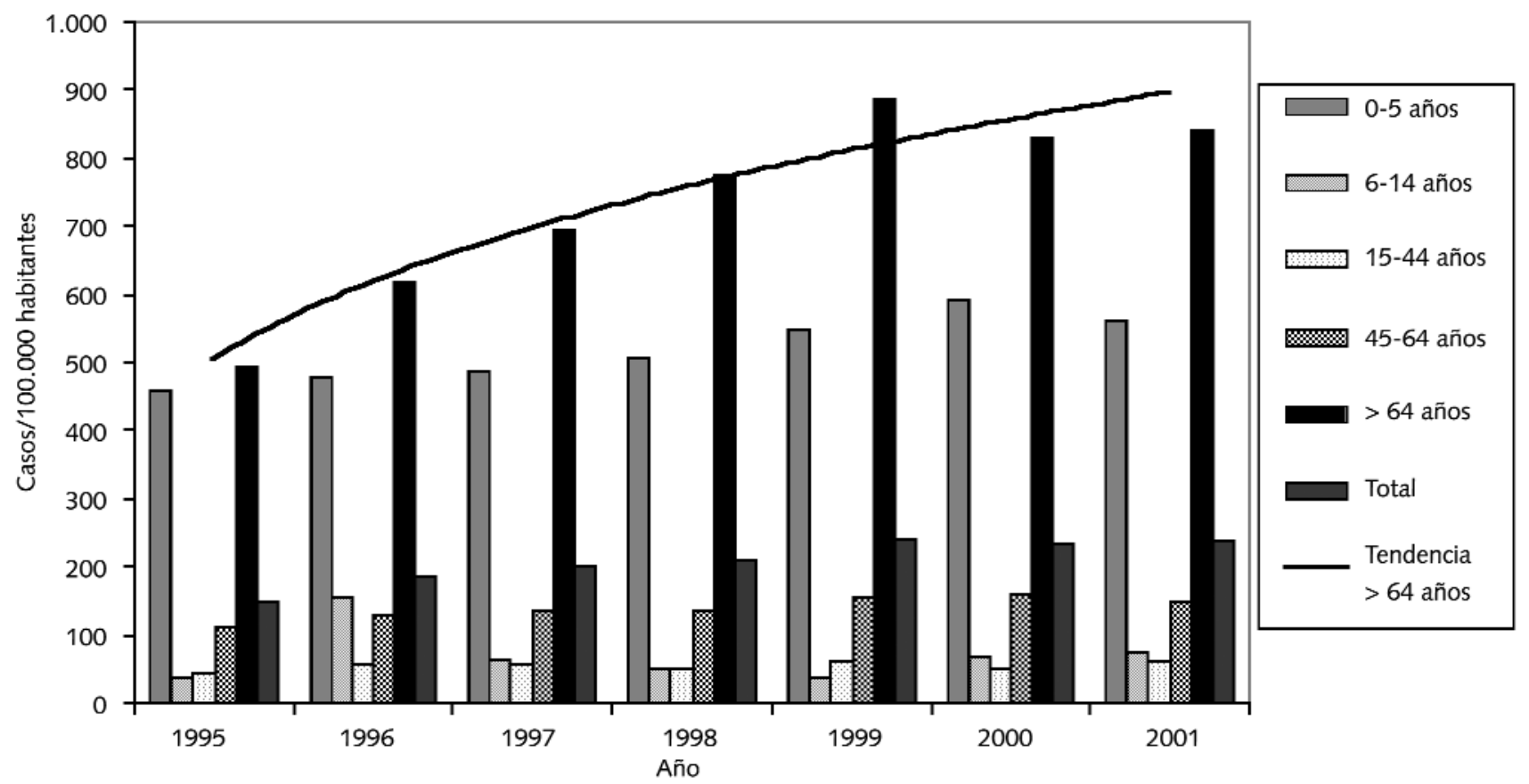

Fig. 1.- Evolución de las incidencias de las neumonías por grupos de edad, durante el periodo 1995-2001. La línea indica la tendencia descrita por el grupo de edad igual o mayor de 65 años.

La incidencia aumenta de manera progresiva desde 1995 hasta 1999, estabilizándose en los años siguientes. El incremento es, estadísticamente significativo $(\mathrm{p}<0,05)$ para los años 1995, 1996, 1997, 1998 y 1999. La evolución de las incidencias en el periodo se muestra en la Figura 1.

\section{Por géneros}

La incidencia es significativamente superior en hombres que en mujeres. Para el conjunto del periodo, la incidencia en hombres es de $268 \pm 2,33$ mientras que en mujeres es de $152 \pm$ 2,01. En la Tabla I y en la Figura 2, se puede observar que a lo largo de todo el periodo de estudio la incidencia es aproximadamente dos veces mayor en hombres que en mujeres. Lo mismo sucede en cada grupos de edad, destacando el grupo de los mayores de 65 años, en los que la incidencia global por género es de $1.088 \pm 14,73 / 100.000$, para hombres y de $483 \pm$ 8,42 para mujeres (Tabla II).

\section{Por edades}

Las edades que más casos registraron durante todo el periodo, fueron: 1 año (2.317 casos, el 3,9\% de los casos totales), 76 años (1.613 casos o el $2,7 \%$ de los casos) y 77 años ( 1.608 casos o el $2,7 \%$ de los casos).

La edad media global fue de 56,7 años, distinguiendo para los mayores de 65 años, una edad media de 77,6 años (D.s. 7,41 ) siendo en hombres de 76,8 (D.s. 7,16) y en mujeres de 78,9 (D.s. 7,63).

Por grupos de edad, el 13,7\% de los casos fueron del G1, el 2,8\% del G2, el 12,1\% del G3, el 14,9\% del G4 y el 56,6\% para los de 65 o más años. En la Tabla II, podemos ver las incidencias por grupos de edad y su evolución a lo largo del periodo.

\section{Por provincias}

Se registraron en la provincia de Valencia el $56,5 \%$ de los casos, el 28,7\% en Alicante y el 9,1\% en Castellón y un 5,7\% de casos perdidos.

La incidencia fue de $219 \pm 2,35 / 100.000$, para Valencia, siendo de $279 \pm 3,80$, para hombres y de $162 \pm 2,83$ para mujeres. En la provincia de Alicante la incidencia fue de 173 $\pm 2,60 / 100.000$, siendo de $224 \pm 4,21$, para hombres y de 125 $\pm 3,09$, para mujeres. En la provincia de Castellón, la incidencia fue de $166 \pm 4,42 / 100.000$, siendo $215 \pm 7,17$ para hombres y $118 \pm 5,24$ para mujeres. Esto nos indica que existen diferencias estadísticamente significativas entre los valores de la incidencia para la provincia de Valencia respecto de Alicante y Castellón.

En la provincia de Valencia, la edad media de los casos fue de 57 años (D.s. 29,0) siendo de 57,34 años en hombres y 56,54 años en mujeres, (D.s. de 27,6 y 31,16 respectivamente). En la provincia de Alicante, la edad media fue de 56,3 años (D.s. 28,85), siendo de 56,88 años en hombres y 55,29 años en mujeres, (D.s. 27,58 y 30,89 respectivamente). En Castellón la edad media fue de 55,17 años (D.s. 29,97) siendo de 56,38 años en hombres y 52,99 años en mujeres (D.s. 28,50 y 32,34 respectivamente).

En mayores de 65 años, en Valencia, la edad media fue de 77,83 años (D.s. 7,48) siendo de 76,91 años en hombres y 


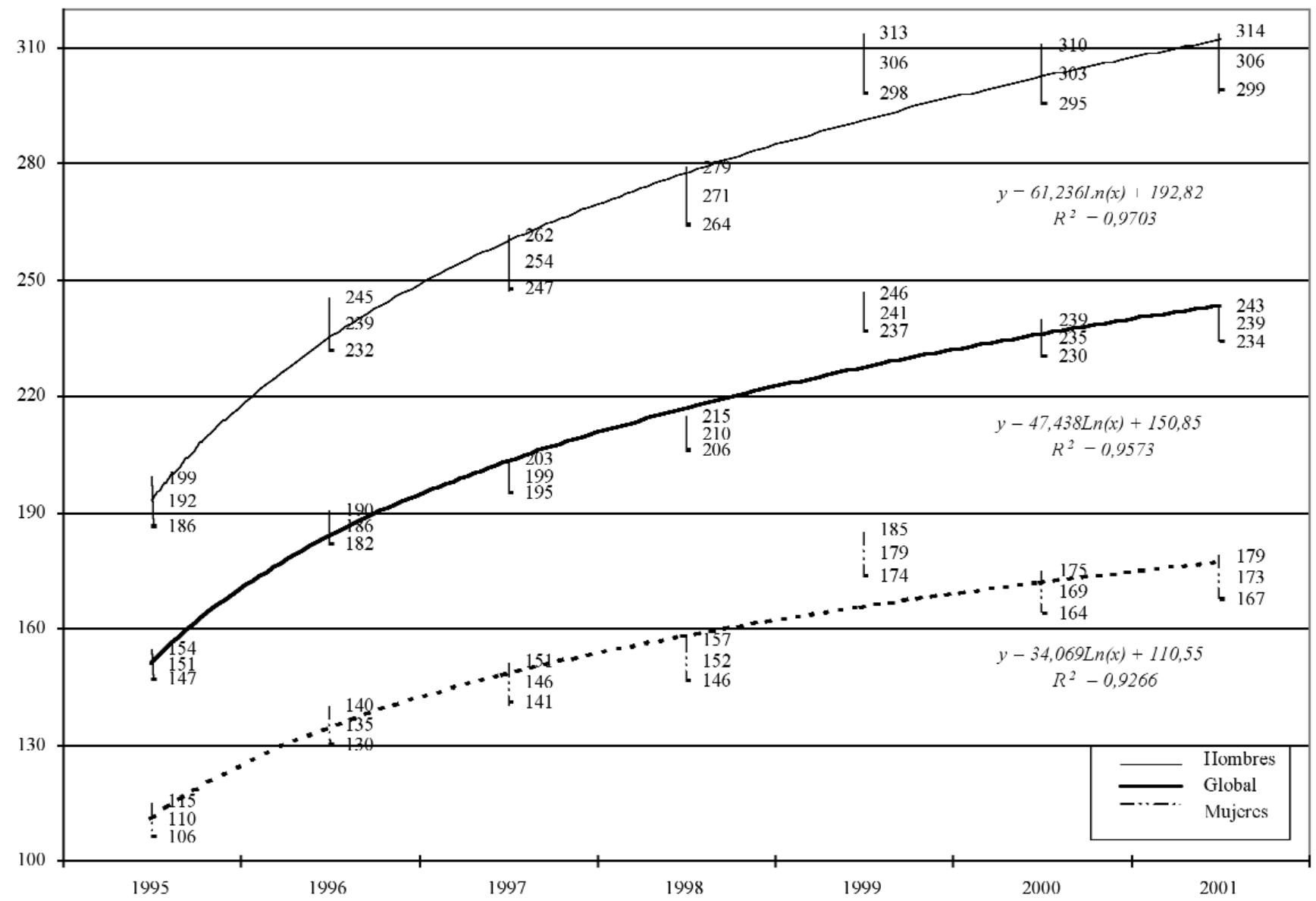

Fig. 2.- Evolución de las neumonías a lo largo del periodo de estudio. Evidencia gráfica de las diferencias significativas existentes entre géneros respecto de los distintos años del periodo de estudio. Casos por 100.000 y año.

\section{TABLA II}

REGISTRO DE LAS INCIDENCIAS POR AÑO, POR GRUPOS DE EDAD, POR GÉNEROS Y TOTALES. CALCULADAS POR 100.000 HABITANTES

\begin{tabular}{lrrrrrrrrrr}
\hline & \multicolumn{1}{c}{ Años } & \multicolumn{3}{c}{ Género } \\
\hline Grupos de edad & 1995 & 1996 & 1997 & 1998 & 1999 & 2000 & 2001 & Hombres & Mujeres & Total \\
\hline 0-5 años & 458 & 480 & 487 & 507 & 548 & 591 & 560 & 561 & 471 & 517 \\
6-14 años & 37 & 156 & 64 & 52 & 38 & 66 & 76 & 60 & 58 & 59 \\
15-44 años & 44 & 57 & 57 & 50 & 611 & 52 & 61 & 71 & 37 & 54 \\
45-64 años & 111 & 128 & 135 & 137 & 157 & 160 & 149 & 201 & 81 & 140 \\
$>$ 64 años & 492 & 620 & 695 & 775 & 888 & 830 & 839 & 1.088 & 483 & 740 \\
Total & 151 & 186 & 199 & 210 & 241 & 235 & 238 & 268 & 152 & 209 \\
\hline
\end{tabular}

79,31 años en mujeres, (D.s. de 7,22 y 7,64 respectivamente). En Alicante fue de 77,33 años (D.s. 7,31) siendo de 76,67 años en hombres y 78,45 años en mujeres, (D.s. 7,08 y 7,56 respectivamente). En Castellón fue de 77,43 años (D.s. 7,1) siendo de 76,9 años en hombres y 78,28 años en mujeres, (D.s. 6,9 y 7,4 respectivamente).

\section{Estancias medias}

En los hospitales de la Comunidad Valenciana, la estancia media de ingreso por neumonía, es de 10,9 días, oscilando entre 6,7 días en el G1 y 13,5 días en el G4, no mostrando diferencias significativas entre las variables estudiadas, en cuanto a la duración del ingreso (Fig. 3). El total de estancias por año de estudio y las estancias medias quedan reflejados en la Tabla III.

En cuanto a las estancias medias de hospitalización por neumonía, exclusivamente o acompañada de otro diagnóstico, se ha podido constatar que prácticamente el tiempo es el mismo, ya se trate de una neumonía por neumococo o por cualquier otra causa. 


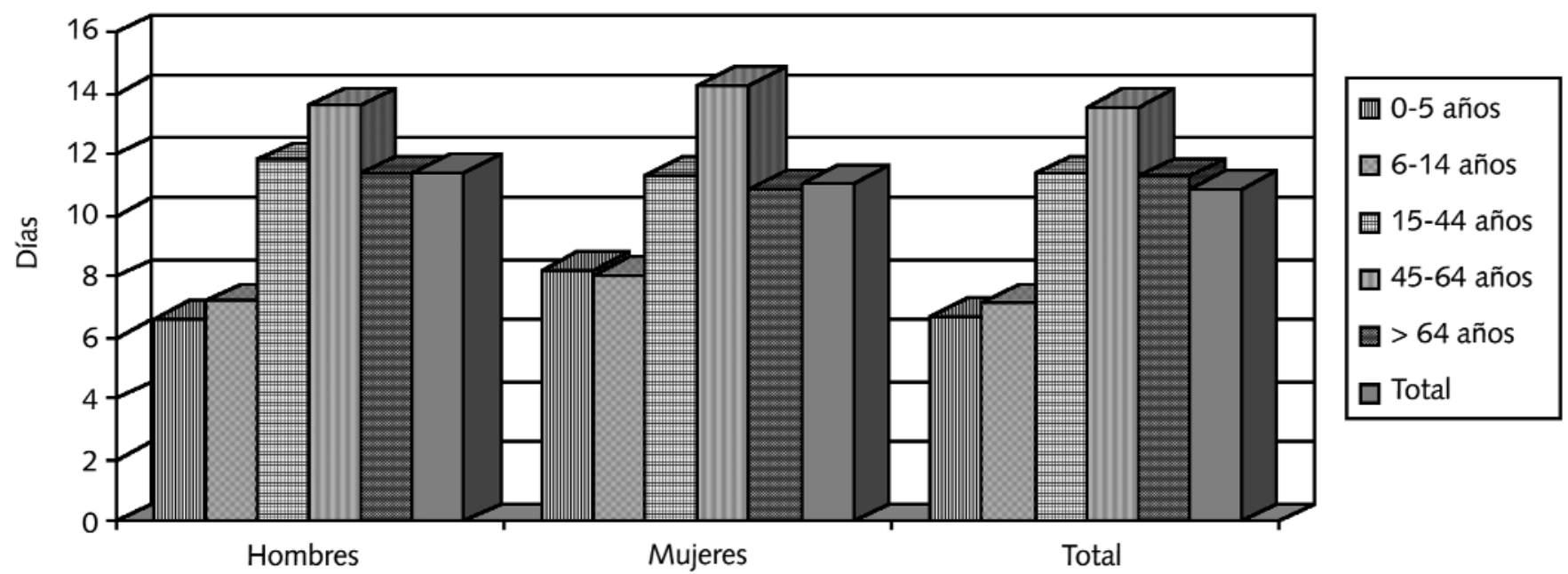

Fig. 3.- Representación gráfica de la estancia media en los hospitales públicos de la Comunidad Valenciana por neumonía, distinguiendo géneros y totales.

TABLA III

NÚMERO DE DÍAS TOTALES DE HOSPITALIZACIÓN POR NEUMONÍA, POR AÑO DE ESTUDIO. ESTANCIAS MEDIAS CALCULADAS PARA EL PERIODO Y POR AÑOS, SEPARANDO GÉNEROS Y GRUPOS DE EDAD

\begin{tabular}{|c|c|c|c|c|c|c|c|c|c|}
\hline \multirow[t]{3}{*}{ Año } & \multirow{3}{*}{$\begin{array}{c}\text { Número de } \\
\text { estancias }\end{array}$} & \multicolumn{2}{|l|}{ Género } & \multicolumn{5}{|c|}{ Estancias medias por grupo de edad } & \multirow{3}{*}{$\begin{array}{l}\text { Total } \\
\text { ( } \bar{x} \text { días })\end{array}$} \\
\hline & & Hombres & Mujeres & $0-5$ & $6-14$ & $15-44$ & $45-64$ & $>64$ & \\
\hline & & ( $\bar{X}$ días $)$ & ( $\bar{X}$ días) & años & años & años & años & años & \\
\hline 1995 & 77.451 & 12,7 & 11,4 & 7,2 & 7,6 & 12,2 & 15,7 & 13,1 & 12,2 \\
\hline 1996 & 88.175 & 11,9 & 11,4 & 6,8 & 7,1 & 12,4 & 15,0 & 12,5 & 11,7 \\
\hline 1997 & 90.117 & 11,4 & 10,8 & 7,6 & 7,8 & 11,1 & 13,5 & 11,7 & 11,2 \\
\hline 1998 & 93.793 & 11,2 & 10,8 & 6,5 & 7,2 & 12,1 & 13,3 & 11,5 & 11,0 \\
\hline 1999 & 103.548 & 10,7 & 10,2 & 6,5 & 8,3 & 10,9 & 12,7 & 10,7 & 10,5 \\
\hline 2000 & 99.749 & 10,5 & 9,9 & 6,4 & 6,9 & 11,1 & 12,8 & 10,5 & 10,3 \\
\hline 2001 & 101.303 & 10,4 & 9,6 & 6,1 & 6,2 & 10,1 & 12,5 & 10,5 & 10,1 \\
\hline Total & 651.136 & 11,1 & 10,5 & 6,7 & 7,2 & 11,3 & 13,5 & 11,3 & 10,9 \\
\hline
\end{tabular}

\section{DISCUSIÓN}

Las neumonías en todas sus formas constituyen una importante causa de morbilidad hospitalaria (10) y generan un número importante de estancias en la Comunidad Valenciana. Con los datos del CMBD, se ha podido ver la evolución que ha descrito a lo largo de 7 años la incidencia, de la enfermedad neumónica que requiere hospitalización. Esta incidencia es de 209 por 100.000 habitantes. Las cifras se incrementan significativamente de año en año, estabilizándose en los años 1999, 2000 y 2001.

Las tasas de incidencia no coinciden con los apuntados por algunos autores (11-15), que registran incidencias muy inferiores a los nuestros debido a que las poblaciones y el ámbito son diferentes y porque nosotros hemos estudiado todas las neumonías (bronconeumonía, neumonía por organismo no especificado y neumonía neumocócica) y la mayor parte de la literatura se refiere exclusivamente a las neumonías neumocócicas.
Nosotros hemos estimado para el conjunto de neumonías neumocócicas (un $14,11 \%$ de los casos) una incidencia de 29 por 100.000 habitantes, siendo estos datos objeto de otro artículo (22) pero que son muy similares al 27,9 que apuntan Domínguez et al. para el S. pneumoniae (16).

Por géneros, encontramos que las incidencias son mayores en hombres que en mujeres, con una razón de 1,7 para el conjunto de la Comunidad. En el grupo de mayores de 64 años, es donde esta diferencia es más acusada con una razón hombres/mujeres de 2,25, coincidiendo con los datos citados para Cataluña (17).

La incidencia en los mayores de 64 años del 740 por 100.000, para todo el periodo estudiado muestra además un evidente incremento a lo largo de los años analizados, llegando a ser del doble en este grupo de edad. Mieckowski y cols. (18), justifica estos datos por el envejecimiento de la población en las sociedades industrializadas como la nuestra.

Butler (19) y otros autores encuentran $(10,20)$, como nosotros, una mayor incidencia en los grupos de edad mas extremos de la vida (menores de 5 años y mayores de 65). 
En el análisis territorial por provincias encontramos que existen diferencias observadas tanto en las incidencias (oscilando entre 166 de Castellón y 219 de Valencia) como en la edad media con que se ingresa por neumonía en nuestros hospitales (oscilando entre 55,7 en Castellón y 57 años en Valencia), y concluimos que existen diferencias significativas y que cabría profundizar en el análisis de otras variables para poder establecer otras hipótesis como la organización de los recursos o las inherentes a la localización geográfica.

El número de estancias en los centros hospitalarios para el periodo fue de 651.136 , sin producirse grandes variaciones a lo largo de los años estudiados. Cuando se estudia por géneros, la razón hombre/mujer se muestra de nuevo y los resultados en número de estancias son casi la mitad en mujeres, siendo el grupo de los mayores de 64 años los que mayor número de estancias generaron. Esto reflejaría la mayor incidencia en estos grupos de población.

Las diferencias encontradas en los distintos grupos de edad en la estancia media no son estadísticamente significati-

\section{Bibliografía}

1. WHO, Pneumococcal vaccines. Wkly Epidemiol Rec. 1999; 74: 177-84

2. Fenoll Comes A, Casal Lombos J, Estudio de los serotipos de Streptococcuss pneumoniae en España. En: Moraga Llop, EA (Ed). La enfermedad neumocócica en el niño. Prous Science, Barcelona 2001; 55-67.

3. Centers for Disease Control and Prevention. Recommendations of the Advisory Committee on Immunization Practices (ACIP): prevention of Pneumococcal Disease. MMWR 1997; 46 (RR-8): 1-24.

4. Centers for Disease Control and Prevention. Preventing pneumococcal disease among infants and young children: Recommendations of the Advisory Comettee on Immunization Practices (ACIP). MMWR 2000; 49 (No. RR-9): 1-35.

5. MUSHER D. Streptococcuss pneumoniae. En: Mandell GL., Bennett JE. \& DOLIN R. Principles and Practice of infectious Diseases, 5th ed. Churchill Livingstone, Philadelphia 2000; 2128-2146.

6. www.vacunas.net. Guía Práctica de Vacunaciones

7. Casado Flores J, Aristegui J, Rodrigo de Liria C, Martinón JM, Hernández Pérez C, y grupo para el Estudio de Meningitis Neumocócica. Prevalencia de la Meningitis neumocócica en niños españoles. An Esp Pediatr 2002; 56: 5-9.

8. Proyecto EPINE, Estudio de Prevalencia de Infecciones Nosocomiales en España, 1990-99. Evolución de la Prevalencia de las Infecciones Nosocomiales en los Hospitales Españoles. En: Vaque J. Roselló J. Editores. Sociedad Española de Medicina Preventiva, Salud Pública e Higiene. Madrid, 2001. IM\&C, S.A.

9. www.ine.es

10. Torres A, Soler N. Evaluación de la etiología y del abordaje terapéutico de la neumonía adquirida en la comunidad. Med Clin (Barc) 2001; 116: $179-81$.

11. Batalla J, Urbiztondo L, Ciruela P, Martínez M, Boldú M. Cobertura vacunal antineumocócica en la población de 65 años de Cataluña. En: Campins Martí-Moraga Llop, EA (Ed). Vacunas 2002. Prous Science, Barcelona 2002; 173-180.

12. Ament A, Baltussen R, Duru G, Rigaud-Bully C, De Graeve D, Ortqvist vas aunque su rango oscile entre 6,7 y 13,5 días, siendo el grupo de edad entre 45 y 64 años el que presenta una estancia media mayor aunque esta ha ido disminuyendo hasta estabilizarse en los últimos años del periodo estudiado. Hecho que podría deberse a la variabilidad clínica o a la evolución de la práctica y que dispara los costes por la enfermedad (21).

Queda por analizar del conjunto de ingresos por neumonía cual es la incidencia de las debidas al S. pneumonie, exclusivamente.

Algunas hipótesis que quedan por contrastar después de los datos aportados en este trabajo es ver si las incidencias disminuirán en los años siguientes, como consecuencia de las actuaciones de vacunación en población mayor de 64 años con la vacuna de 23 polisacáridos puesta en marcha por la Consellería de Sanidad de Valencia a partir del año 2002. Así como las causas que expliquen las diferencias en la incidencia por géneros o procedencia, el cambio de tendencia en la evolución de las mismas a lo largo del periodo o la mayor estancia media en el grupo de edad de 45 a 64 años.
A, et al. Cost-effectiveness of pneumococcal vaccination of older people: a study in 5 western European countries. Clin Infect Dis 2000; 31 : 444-450.

13. Vlasich C. Pneumococcal infection and vaccination in the elderly. Vaccine 2001; 19: 2233-2237.

14. Cornu C, Yzebe D, Leophonte P, Gallita J, Boissel JP, Cucherat M. Efficacy of pneumococcal polysacharide vaccine in immunocompetent adults: a meta-analysis of randomized triasl. Vaccine 2001; 19: 2233 2237.

15. Espin MI, Sandoval A, Ruiz J, Navarro JA, García J, Pérez Flores D. Enfermedad neumocócica invasiva en niños de la Región de Murcia. Gac Sanit 2002; 16: 385-391.

16. Dominguez A, Salleras L, Cardeñosa N, Ciruela P, Carmona G, Martínez A, et al. The epidemiology of invasive Streptococcus pneumoniae disease in Catalonia (Spain). A hospital-based study. Vaccine 2002; 20: 2989-2994.

17. Domínguez A, Salleras L. Epidemiología de la enfermedad invasora por neumococo. En: Campins Martí-Moraga Llop, EA (Ed). Vacunas 2002. Prous Science, Barcelona 2002; 163-173.

18. Mieckowski T, Wilson S. Adult pneumococcal vaccination: a review of physician and patient barriers. Vaccine 2002; 20: 1383-1392.

19. Butler JC, Schuchat A. Epidemiology of pneumococcal infections in the elderly. Drugs Aging 1999; 15: 11-9.

20. Kim P, Musher DM, Glezen WP, et al. Association of invasive pneumococcal disease with season, atmospheric conditions, air pollution, and the isolation of respiratory viruses. Clin Infect Dis 1996; 22: 100-106.

21. Plans P. 2002. Coste efectividad de la vacunación antineumocócica 23valente en Cataluña. Gac Sanit 2002; 16: 392-400.

22. Comes AM, Lluch JA, Portero A, Pastor E, Sanz M. Incidencia de las neumonías neumococcicas en el ámbito hospitalario de la Comunidad Valenciana durante el periodo 1995-2001. Rev Esp Salud Pública 2004; 78: 481-494. 